\title{
DESEMPENHO DE NOVILHAS DA RAÇA NELORE MEDIANTE O USO DE BETABLUCANAS E PROBIÓTICOS
}

\author{
André Felipe Berto de Almada ${ }^{1}$ \\ Ranufo Piau Júnior ${ }^{2}$ \\ Valmir Fernandes ${ }^{3}$ \\ Jessé Lahos Borges ${ }^{4}$ \\ André Felipe da Silva ${ }^{5}$ \\ João Vitor Moraes Simões ${ }^{6}$ \\ Vanderson dos Santos Silva ${ }^{7}$
}

ALMADA, A. F. B. de; PIAU JÚNIOR, R.; FERNANDES, V.; BORGES, J. L.; SILVA, A. F. da; SIMÕES, J. V. M.; SILVA, V. dos S. Desempenho de novilhas da raça Nelore mediante o uso de betablucanas e probióticos. Arq. Cienc. Saúde UNIPAR, Umuarama, v. 21, n. 3, p, 175-179, set./dez. 2017.

\begin{abstract}
RESUMO: Os aditivos são suplementos que podem contribuir para aumentar o desempenho dos animais, visto que eles podem melhorar a conversão alimentar e o ganho de peso nos animais. As 1,3-1,6 betaglucanas são agentes imunomoduladores com potencial na prevenção e controle de doenças infecciosas, e agem como substrato aos probióticos. O uso de probióticos na alimentação pode melhorar o desempenho dos animais, competindo com bactérias patogênicas e agindo na imunomodulação. O objetivo deste trabalho foi avaliar o efeito do uso de betaglucanas (prebiótico) e probióticos sobre o desempenho de novilhas confinadas da raça Nelore. Sessenta novilhas da raça Nelore com cerca de 24 meses de idade foram distribuídas aleatoriamente formando quatro grupos que receberam prebióticos (PRE), probiótico (PRO), e a simbiose pela associação destes dois aditivos (SIM) e um grupo controle. Os animais sofreram a adaptação durante uma semana, e todos foram identificados com brincos. Nos dias 0,30, 60 e 90 ocorreu a pesagem dos animais e dieta oferecida e suas sobras, para o cálculo do ganho de peso e da conversão alimentar. Não houve diferença estatística para o ganho de peso, entretanto ocorreu um melhor rendimento quanto este parâmetro nos grupos que receberam probióticos e com a simbiose de probióticos e prebióticos. , a simbiose, aparentemente, também proporcionou um aumento no consumo médio diário. Probióticos e a simbiose também aparentam melhorar a conversão alimentar e conversão alimentar da proteína em novilhas confinadas da raça Nelore.
\end{abstract}

PALAVRAS-CHAVE: Confinamento. Conversão alimentar. Ganho de peso. Ganho de peso.

\section{EVALUATION OF BETA-GLUCAN AND PROBIOTICS ON THE PERFORMANCE OF CONFINED NELLORE HEIFERS}

ABSTRACT: Additives are supplements that can contribute to increase the performance of animals. They can improve feed conversion and weight gain. The immunomodulatory agents 1,3-1,6 beta-glucans have the potential to prevent and control of infectious diseases, and also acting as substrate to probiotics. The use of probiotics in feed can improve the performance of the animals, competing with pathogenic bacteria and also acting on immunomodulation. The purpose of this work was to assess the effect of the use of beta-glucan (prebiotic) and probiotics on the performance of confined Nellore heifers. Sixty 24-month-old Nellore heifers were randomly assigned to four groups that received prebiotics (PRE), probiotic (PRO), and symbiosis by the association of these two additives (SIM), as well as a control group. The animals were adapted over the period of one week. All animals were identified with earrings. The cattle were submitted to stress by the usual manipulation of weighing in the corral on days 0,30,60 and 90. Feed offered to the animals and the surplus of such feed were also weighed. The weight gain and feed conversion of heifers were evaluated. No statistical differences were found for weight gain. However, it could be concluded that even though there were no statistical differences in weight gain, a better yield could be observed in the biological weight gain in the animals that were supplemented with probiotics and with the symbiosis of probiotics; prebiotics and symbiosis apparently also provided an increase in the average daily consumption. Probiotics and symbiosis also appear to improve feed conversion and protein feed conversion in confined Nellore heifers.

KEYWORDS: Confinement. Food conversion. Prebiotics. Symbiosis. Weight gain.

\section{Introdução}

Atualmente a pecuária ocupa uma posição de destaque no agronegócio brasileiro, dividindo espaço como uma das principais atividades do setor com produtos como soja, milho e cana-de-açúcar. Segundo projeções de 2015 até 2025 a produção de carne bovina vai subir $23,3 \%$, enquanto o consumo interno vai aumentar $17,8 \%$ e as exportações vão subir 37,4\% (BRASIL, 2015).

O grande potencial produtivo do Brasil pode solucionar um dos maiores problemas que o mundo enfrentará nos próximos anos, como a dificuldade de acesso a alimentos devido o crescimento da população, principalmente em países da Ásia, visto que as perspectivas para 2030 é que a população mundial chegue a 8,5 bilhões, em 2050 alcances 9,7 bilhões, e em 2100 atinja 11 bilhões (ONU, 2015). Apontando a necessidade de produzir cada vez mais e aproveitar melhor os recursos para elevar a produtividade em todo setor agrícola no Brasil, inclusive a pecuária.

Alguns aditivos alimentares, como a monensina, comumente utilizados nas dietas de animais de produção vêm mostrando sinais positivos nos desempenhos de ganho

DOI: 10.25110/arqsaude.v21i3.2017.6193

${ }^{1}$ Mestre e Zootecnista, Endereço: Rua Izaías Antônio Pereira n ${ }^{\circ}$ 127, Naviraí - Mato Grosso do Sul, Email: andrefelipe@zootecnista.com.br

${ }^{2}$ Doutor e Médico Veterinário, Endereço: Rua Marabá, 3322, Zona 1- Umuarama - PR - 87510-100, Email: piau@prof.unipar.br

${ }^{3}$ Mestre e Médico Veterinário, Endereço: Universidade Paranaense - Praça Mascarenhas de Moraes 4282, cx. p. 224, 87502-210, Umuarama - PR, E-mail: veteagro@gmail.com

${ }^{4}$ Mestrando e Médico Veterinário, endereço: Rua Getúlio Vargas no 2385, Alto Piquiri - PR, Email: 1ahos@outlook.com

${ }^{5}$ Graduando em medicina veterinária, Endereço: Av. Mário Marangoni, 1431, Centro, Moreira Sales - PR, Email: andre_acer@hotmail.com

${ }^{6}$ Graduando em medicina veterinária, Praça Mascarenhas de Morais nº 5075, Edifício Antunes, apto. 303 Umuarama - PR, Email: joaovitormorais2009@ hotmail.com

${ }^{7}$ Graduando em medicina veterinária, Sitio Estancia da Amizade, Estrada Tapira para Douradina KM 02, Email: vanderson102015@hotmail.com 
de peso, entretanto alguns cuidados devem ser tomados, pois segundo Nicodemos (2001) o uso de antibióticos exige muito cuidado pois nem sempre é possível suplementar esses aditivos nas dosagens indicadas, e a suplementação abaixo dos níveis recomendados provoca a resistência de algumas bactérias, com destaque para aquelas que são patogênicas aos animais e também ao ser humano.

Além da melhoria no desempenho produtivo que as leveduras proporcionam aos bovinos, pode ocorrer um aumento da população e da atividade dos microrganismos ruminais, além de uma maior proporção do ácido graxo de cadeia curta propionato em relação ao ácido graxo de cadeia curta acetato (HARRISON et al. 1988; GUEDES et al., 2008). Outro benefício é no controle do $\mathrm{pH}$ ruminal, podendo chegar em níveis próximos ou até mesmo superiores ao bicarbonato de sódio, mostrando eficiência como tamponante (MARDEN et al., 2008), demostrando ser uma alternativa válida para dietas de confinamento.

Fale das betaglucanas que está utilizando neste trabalho e do seu efeito imunomodulador, visto que falou disto no resumo.

O objetivo deste trabalho foi avaliar o desempenho produtivo de novilhas da raça Nelore confinadas, consumindo prebiótico e probióticos numa dieta de baixo valor nutritivo.

\section{Material e Métodos}

O experimento foi aprovado pelo Comitê de Ética em Pesquisa Envolvendo Experimentação Animal da Universidade Paranaense, sob o protocolo $n^{\circ} 30166 / 2016$.

O experimento foi realizado na fazenda experimental da Universidade Paranaense (Unipar), situada no município de Umuarama, no estado do Paraná, na rodovia PR 482. No período de 25 de julho de 2016 a 24 de outubro de 2016.

Cada baia possuía $64 \mathrm{~m}^{2}$,o coxo tinha acesso frontal à ração fornecida e a mesma metragem do comprimento da baia. Os bebedouros mediam 1,5 $\mathrm{m}$ de comprimento e $1 \mathrm{~m}$ de largura. Cada baia alojou 15 novilhas, deixando uma média de $4,27 \mathrm{~m}^{2}$ por animal e $0,53 \mathrm{~m}$ de linha de cocho.

Sessenta novilhas da raça Nelore com aproximadamente 24 meses de idade foram distribuídas aleatoriamente em quatro grupos: controle, grupo prebióticos (PRE), grupo probióticos (PRO), e grupo prebióticos e probióticos com a simbiose destes dois bioativos (SIM). Os animais sofreram a adaptação à dieta controle durante uma semana na dieta, e todos foram identificados com brincos.

Pesou-se 40 animais, ou seja, 10 animais de cada grupo, escolhidos por sorteio ao acaso, sendo que as coletas ocorreram nos mesmos animais a cada 30 dias.

Os animais pertenciam ao plantel da fazenda experimental da Universidade Paranaense, onde se encontravam em regime de recria a pasto, e posteriormente foram conduzidas para o sistema de engorda e terminação no manejo de confinamento na fazenda.

A dieta foi fornecida ad libitum, os animais eram arraçoados duas vezes por dia às $08 \mathrm{~h} 00 \mathrm{~min}$ e às $16 \mathrm{~h} 00 \mathrm{~min}$, as sobras foram pesadas, semanalmente, para estabelecimento da média de consumo por animal dia.

As rações tinham como ingredientes gérmen de milho, farelo de trigo, resíduo de mandioca e núcleo mineral
(Tabela1).

Tabela 1: Composição básica da ração fornecida para as novilhas.

\begin{tabular}{lcc}
\hline \multicolumn{1}{c}{ Ingrediente } & $\begin{array}{c}\text { Inclusão na } \\
\text { matéria seca }\end{array}$ & $\begin{array}{c}\text { Inclusão na } \\
\text { matéria natural }\end{array}$ \\
\hline Gérmen de milho & $15,32 \%$ & $6,0 \%$ \\
Farelo de trigo & $47,84 \%$ & $21,0 \%$ \\
Resíduo de mandioca & $28,61 \%$ & $70,0 \%$ \\
Núcleo mineral & $8,23 \%$ & $3,0 \%$ \\
\hline
\end{tabular}

Os níveis de garantia foram medidos nos parâmetros de matéria seca, proteína bruta, fibra detergente neutro e fibra detergente ácido (Tabela 2).

Tabela 2: Composição bromatológica da ração fornecida para as novilhas.

\begin{tabular}{lc}
\hline \multicolumn{1}{c}{ Descrição } & $\begin{array}{c}\text { Valores } \\
\text { encontrados }\end{array}$ \\
\hline Matéria Seca & $59,08 \%$ \\
Proteína Bruta & $8,37 \%$ \\
Fibra Detergente Neutro & $23,25 \%$ \\
Fibra Detergente Ácido & $10,91 \%$ \\
\hline
\end{tabular}

Os aditivos utilizados no experimento foram: 1,31,6 betaglucanas (prebiótico); Ruminobacter amylophilum, Ruminobacter succinogenes, Succinovibrio dextrinosol nens, Bacillus cereus, Lactobacilus acidophilus, Enterococcus faecium, Saccharomyces cerevisiae (probiótico) e a associação destes dois aditivos como simbiótico.

Utilizou-se aditivos orgânicos, fornecidos logo após o trator arraçoar os cochos, para melhorar a mistura e garantir que todos os animais consumissem a quantidade necessária do aditivo (5 gramas de aditivo/animal/dia). Diariamente, eram administrados 3 quilos de uma mistura, cujo veículo era o gérmen de milho, o consumo diário de cada animal na mistura: veiculo + aditivo era de 200 gramas por dia.

- No grupo controle os animais consumiam a dieta normal suplementada com $200 \mathrm{~g}$ de gérmen de milho.

- No grupo prebióticos (PRE) os animais consumiam a dieta normal suplementada com $195 \mathrm{~g}$ de gérmen de milho e $5 \mathrm{~g}$ de prebióticos (1,31,6 betaglucanas).

- No grupoProbióticos (PRO) os animais consumiam a dieta normal suplementada com $195 \mathrm{~g}$ de gérmen de milho e $5 \mathrm{~g}$ de cada animal consumindo então: $1,5 \times 10^{9}$ UFC de Ruminobacter

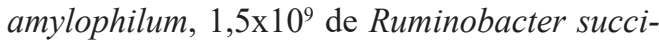

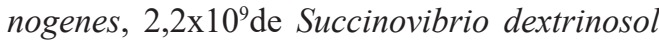
nens, $1,75 \times 10^{9}$ de Bacillus cereus, $1,75 \times 10^{9}$ de

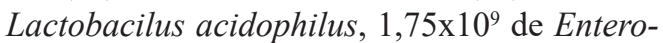
coccus faecium e $3,75 \times 10^{10}$ de Saccharomyces cerevisiae.

- No grupo prebióticos e probióticos (SIM) os animais consumiam a dieta normal suplementada com $190 \mathrm{~g}$ de gérmen de milho, $5 \mathrm{~g}$ de prebióticos e $5 \mathrm{~g}$ de probióticos.

As pesagens ocorreram no início do experimento 
(D0), aos 30 dias (D30), 60 dias (D60) e aos 30 dias (D90).

Os dados do ganho de peso foram analisados no programa Bioestat 5.3 pelo teste TUKEYa ao com nível de significância de 5\% (AYRES et al., 2007).

\section{Resultados e Discussão}

O peso médio inicial das novilhas do presente estudo foi de 282,1 kg para o grupo controle, $306,4 \mathrm{~kg}$ para o grupo PRE, 304,4 kg para o grupo PRO e $263,5 \mathrm{~kg}$ para o grupo SIM, iniciando com diferença estatística entre os grupos PRE e PRO com o grupo SIM (Tabela 3).

Tabela 3: Média \pm erro padrão do peso de novilhas (g) confinadas durante 90 dias.

\begin{tabular}{lllll}
\hline \multirow{2}{*}{$\begin{array}{c}\text { Tempo de } \\
\text { Avaliação }\end{array}$} & \multicolumn{1}{c}{ Controle } & \multicolumn{1}{c}{ PRE } & \multicolumn{1}{c}{ PRO } & SIM \\
\cline { 2 - 5 } & \multicolumn{1}{c}{ PRUPOS } \\
\hline D0 & $283.3000 \pm 6,0920^{\mathrm{ab}}$ & $306.4000 \pm 657,1168^{\mathrm{a}}$ & $304.4000 \pm 6,1086^{\mathrm{a}}$ & $263.5000 \pm 56,1305^{\mathrm{b}}$ \\
D30 & $295.2000 \pm 6,387672^{\mathrm{ab}}$ & $318.5000 \pm 9,9680^{\mathrm{a}}$ & $319.4000 \pm 768,1978^{\mathrm{a}}$ & $284.7000 \pm 6,21717^{\mathrm{b}}$ \\
D60 & $303.6000 \pm 7,157355^{\mathrm{ab}}$ & $324.5000 \pm 9710,2385^{\mathrm{ab}}$ & $332.5000 \pm 8,0970^{\mathrm{a}}$ & $296.4000 \pm 67,0304^{\mathrm{b}}$ \\
D90 & $310.9000 \pm 6,7880^{\mathrm{ab}}$ & $332.3000 \pm 8,03839^{\mathrm{ab}}$ & $341.0000 \pm 10965135^{\mathrm{a}}$ & $302.0000 \pm 7,91076^{\mathrm{b}}$ \\
\hline
\end{tabular}

Letras diferentes na linha demonstram diferença estatística entre grupos pelo teste Tukey.

Não houve diferença estatística entre os grupos controle e os experimentais (PRE e PRO), assim como identificado por Lima et al. (2003) e Appelt et al. (2010) em experimentos com aves que não encontraram resultados significativos no desempenho de ganho de peso de frangos de corte fazendo uso de probióticos na sua dieta de frangos de corte. Entretanto, resultados diferentes foram encontrados por Silva et al. (2009) e Silva et al. (2010) observaram um um melhor ganho de peso em frangos nas fases iniciais em condições de temperaturas desfavoráveis a sua produtividade quando utilizou-se com uso de prebióticos em sua dieta para frangos nas fases iniciais, em que proporcionou um melhor ganho de peso nas aves em condições de temperaturas desfavoráveis a sua produtividade.

Bittar et al. (2016) não encontraram diferença estatística no ganho de peso de bezerros Bos taurus fazendo uso de um pool de bactérias benéficas ao rúmem; enquanto Rasteiro et al. (2007) e Schuz Neto et al. (2013) identificaram que afirmam que o uso de probióticos na dieta de ruminantes proporcionouam um melhor desempenho produtivo na engorda. Por outro lado, Magnabosco et al. (2010) apontam uma melhora no desempenho em produção de leite, utilizando uma simbiose de prebióticos e probióticos na alimentação de vacas leiteiras em Goiás, resultados que podem ser comparados ao bom desempenho em ganho de peso do grupo SIM.

Os animais do grupo PRO, que ingeriam uma dieta de $8,39 \%$ de proteína bruta teve uma média diária de ganho de peso de $407 \mathrm{~g}$, e os animais do grupo SIM obtiveram 428 $\mathrm{g}$, sugerindo que tais valores menores que àqueles dos confinamentos convencionais, ocorreram devido à presença do resíduo de mandioca na ração (tabela 1) e dos baixos níveis nutricionais (tabela 2). Segundo o NRC (1984), novilhas com um peso de $300 \mathrm{~kg}$ atingem uma média de ganho de peso diário de $500 \mathrm{~g}$ por dia ao consumirem uma dieta com $8,5 \%$ de proteína bruta, enquanto novilhas de $250 \mathrm{~kg}$ de peso devem consumir uma ração com $8,9 \%$ de proteína bruta para obter uma média de $500 \mathrm{~g}$ por dia. Na figura 2, consideramos cada peso inicial como $100 \%$, os grupos que obtiveram os melhores rendimentos em ganho de peso foram os grupos SIM e PRO (figura 2).

Figura 1: Frequência relativa do ganho de peso de novilhas (g) confinadas durante 90 dias.

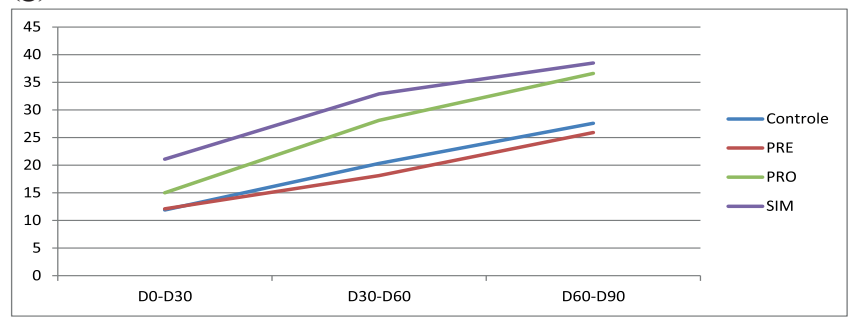

O grupo PRE teve o menor desempenho, mesmo quando comparado com todos os grupos, inclusive o controle, no entanto, quando associado com probióticos (grupo SIM) proporcionam resultados positivos em ganho de peso em novilhas Nelore confinadas com dietas de baixo valor nutricional.

Fatores como consumo e conversão alimentar são importantes para o melhor entendimento do rendimento real do confinamento (Tabela 4).

Não foi possível observar uma diferença representativa no CMD entre os grupos, no que tange ao \%CMD/PV o grupo SIM teve um percentual maior de consumo em relação ao seu peso vivo. Para CA e CA/PB o grupo SIM alcançou as melhores médias, seguido pelo grupo PRO, o grupo PRE apresentou a pior média para estes quesitos.quesitos.

Tabela 4: Média \pm erro padrão do consumo médio diário(CMD), porcentagem do consumo médio diário (CMD/PV), conversão alimentar (CA) e conversão alimentar da proteína (CA/PB) em novilhas confinadas durante 90 dias.

\begin{tabular}{lcccc}
\hline \multicolumn{1}{c}{ GRUPOS } & CMD & \%CMD/PV & CA & CA/PB \\
\hline Controle & $5,1338 \pm 0,2338$ & $1,81 \%$ & 14,39 & 1,49 \\
PRE & $5,1272 \pm 0,2348$ & $1,68 \%$ & 17,82 & 1,84 \\
PRO & $5,2450 \pm 0,2527$ & $1,69 \%$ & 12,90 & 1,33 \\
SIM & $5,2753 \pm 0,2611$ & 1,95 & 12,33 & 1,27 \\
\hline
\end{tabular}


Kuss et al. (2009) observaram que os valores do consumo médio diário de novilhos foram similares entre o grupo com probióticos e os demais grupos. No entanto, as médias encontradas por os dados deste estudo foram inferiores aos identificados por Kuss et al. (2009) foram superiores comparadas as médias do presente estudo.para o \% $\% \mathrm{MD} / \mathrm{PV}$ e superiores quanto ao CA.

Figura 2: Comparativo entre conversão alimentar (CA) e conversão alimentar da proteína bruta $(\mathrm{CA} / \mathrm{PB})$ das novilhas confinadas da raça Nelore entre os grupos.

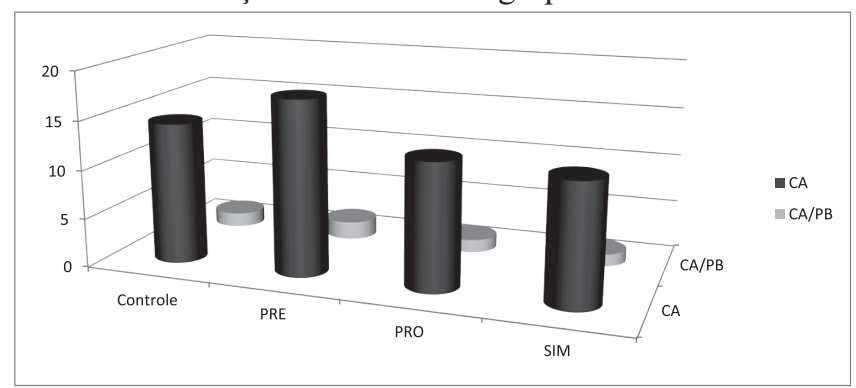

Figura 3: Comparativo da conversão alimentar (CA) e conversão alimentar pela proteína bruta $(\mathrm{CA} / \mathrm{PB})$ entre os grupos.

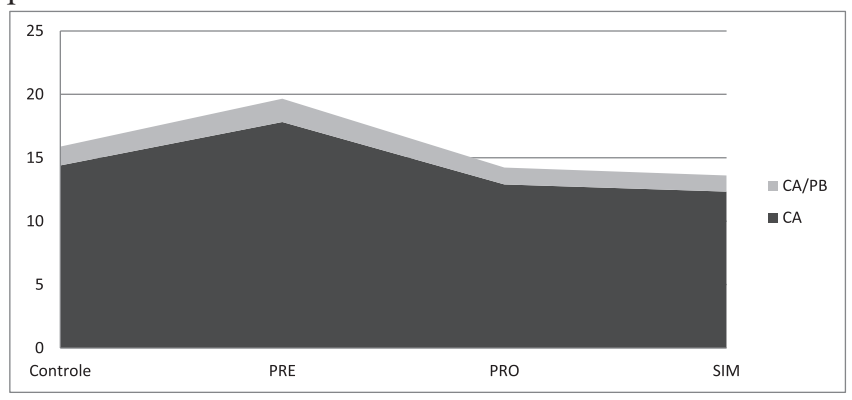

$\mathrm{O}$ aproveitamento da dieta total na $\mathrm{CA}$ e o aproveitamento da proteína na $\mathrm{CA} / \mathrm{PB}$ apresentaram similaridades entre os grupos, conforme pode ser observado na figura 3 , aparentando que a restrição do consumo de proteína afetou a CA e o ganho de peso.

\section{Conclusão}

Mesmo não havendo diferença estatística entre os grupos quanto o ganho de peso, houve um melhor rendimento em ganho de peso biológico nos animais que foram suplementados com probióticos e com a simbiose de probióticos e prebióticos, a simbiose, aparentemente, também proporcionou um aumento no consumo médio diário. Probióticos e a simbiose também aparentam melhorar a conversão alimentar e conversão alimentar da proteína em novilhas confinadas da raça Nelore.

\section{Referências}

APPELT, M. D. et al. Níveis de probiótico em ração de origem animal e vegetal para frangos de corte. Revista Brasileira de Zootecnia, v. 39, n. 4, p. 765-771, 2010.

AYRES, M. et al. (2007). BIOESTAT - Aplicações estatísticas nas áreas das ciências bio-médicas. Ong
Mamiraua, Belém, PA.

BITTAR, C. M. et al. Desempenho e parâmetros sanguíneos de bezerros em sistemas de desaleitamento precoce suplementados com probióticos de bactérias ruminais. Revista Brasileira de Saúde Animal, v. 17, n. 2, p. 249261, 2016.

BRASIL. Ministério da Agricultura Pecuária e Abastecimento. Assessoria de Gestão Estratégica. Projeção do Agronegócio - Brasil 2014/2015 a 2024/2025. Brasília: AGE, 2015. 133 p.

GUEDES, C. M. et al. Effects of a Saccharomyces cerevisiae yeast on ruminal fermentation and fibre degradation of maize silages in cows. Animal Feed Science and Technology, v. 145, p. 27-40, 2008.

HARRISON, G. A. et al. Influence of addition of yeast culture supplement to diets of lactating cows on ruminal fermentation and microbial populations. Journal of Dairy Research, v. 71, qual o número,n. 11, p. 2967-2975, 1988.

KUSS, F. et al. Desempenho e características da carcaça e da carne de novilhos não-castrados alimentados com ou sem adição de monensina e/ou probióticos à dieta. Ciência Rural, v. 39, n. 4, p. 1180-1186, 2009.

LIMA, A. C. F. et al. Efeitos do uso de probióticos sobre o desempenho e atividades de enzimas digestivas de frangos de corte. Revista Brasileira de Zootecnia, v. 32, n. 1, p. 200-207, 2003.

MAGNABOSCO, C. U. et al. Efeito da adição de probióticos e prebióticos na quantidade e qualidade do leite de vacas da raça girolando no bioma cerrado. Comunicado Técnico - Embrapa, v. 165, p. 1-6, 2010.

MARDEN, J. P. et al. How does live yeast differ from sodium bicarbonate to stabilize ruminal $\mathrm{pH}$ in high-yielding dairy cows? Journal of Dairy Science, v. 91, n. 9, p. 3528$3535,2008$.

NICODEMO, M. L. F. Uso de aditivos na dieta de bovinos de corte. Documentos / Embrapa Embrapa Grado de Corte, v. 1517-3747, n. 106, p. 1-54, 2001.

\section{NATIONAL RESEARCH COUNCIL - NRC. Nutrient} Requeriment of beef cattle. Washington, $1984 \mathrm{p}$.

ONU - ORGANIZAÇÃO DAS NAÇÕES UNIDAS. Centro Regional de Informações das Nações Unidas. ONU Projeta que População Mundial Chegue a 8,5 mil milhões em 2030. Brasil. ONU/UNRIC, 2015. 1p.

RASTEIRO, V. S. et al. Adição de probiótico na mistura mineral eleva o ganho de peso de bovinos no período da seca. Arch. Latinoam. Prod. Anim, v. 15, n. 3, p. 83-87, 2007.

NETO, C. S. et al. Efeito da suplementação com probióticos em relação à suplementação com mistura mineral no ganho 
de peso de garrotes Nelore a pastejo extensivo de Panicum maximim. Revista Cientifica Eletrônica de Medicina Veterinária, v. 20, n. 1, p. 1-16, 2013.

SILVA, V. K. et al. Desempenho de frangos de corte de 1 a 21 dias de idade alimentados com rações contendo extrato de leveduras e prebióticos e criados em diferentes temperaturas. Revista Brasileira de Zootecnia, v. 38, n. 4, p. 690-696, 2009.

SILVA, V. K. et al. Yeaste extract and prebiotic in preinitial phase diet for broiler chickens raised under different temperatures. Revista Brasileira de Zootecnia, v. 39, n. 1, p. 165-174, 2010.

Recebido em: 15/06/2017

Aceito em: 29/09/2017 